\title{
UNA EXPERIENCIA EN LA FORMACIÓN HUMANA Y LA INTEGRACIÓN DE SABERES
} María Julia Sierra*

\begin{abstract}
RESUMEN: Los cursos de Problemas de la civilización contemporánea que el Departamento de Estudios Generales del ITAM ofrece a los estudiantes intentan configurar académicamente algunas formas de comprender la realidad actual. Pero también es una apuesta por un marco teórico interdisciplinar, un método dialógico y el entendimiento de que la universidad es la conciencia crítica de la sociedad. Así, se invita a los estudiantes a valorar su responabilidad social.
\end{abstract}

PALABRAS CLAVE: Formación humana, integración de saberes, Estudios generales, Problemas de la civilización contemporánea, humanismo.
ABSTRACT: The courses, Problems of Contemporary Civilization, given by ITAM's General Studies Department, attempt an academic approach to understanding our current reality. They constitute a venture to create an interdisciplinary theoretical framework based on dialogic teaching and the view of university as society's critical conscience. Moreover, these courses entice students to value their social responsibility.

KEYWORDS: formation, integration of knowledge, General Studies, Problems of the Contemporary Civilization, humanism. 


\section{UNA EXPERIENCIA EN LA FORMACIÓN HUMANA Y LA INTEGRACIÓN DE SABERES*}

El hombre es grande en cuanto se conoce miserable. Es demasiado peligroso hacer ver al hombre hasta qué punto es semejante a las bestias, sin mostrarle su grandeza. Y es igualmente demasiado peligroso hacerle ver su grandeza sin su miseria. Es todavía más peligroso dejarle ignorar lo uno y lo otro, pero es muy beneficioso presentarle ambas cosas.

En una palabra, el hombre conoce que es miserable. Es, pues, miserable, por lo que es; pero es grande, porque lo conoce.

¿Qué quimera es, pues, el hombre! ¡Qué maldad! ¿Qué caos! ¡Qué sujeto de contradicciones! Juez de todas las cosas, gusano imbécil - depositario de lo verdadero-acervo de incertidumbres, monstruo incomprensible.

Gloria y desecho. ¿Quién esclarecerá esta confusión? [...] Reconoced pues soberbios qué paradoja sois para vosotros mismos. ¡Humillaos, razón impotente! Callad naturaleza imbécil. Sabed pues, que el hombre supera infinitamente al hombre.

Blaise Pascal**

\section{Preámbulo}

La cuestión primera que subyace en el fondo de los Estudios Generales en el ITAM es la preocupación por el hombre. ¿Quién soy? ¿A dónde voy? ¿Qué quiero? ¿Qué significa ser hombre hoy? Las preguntas no son

* Este artículo es una versión más extensa de la conferencia "Preocupación por el hombre, el mundo y el futuro como tarea. Una experiencia en la formación integral", dictada en la Universidad de Puerto Rico, recinto Río Piedras, el 7 de noviembre de 2013, en el V Simposio Internacional de Estudios Generales.

** Blaise Pascal, Pensamientos, 1981, Madrid, Alianza, pp. 49-50 y 53-4. interrogantes teóricas, son existenciales. Nos interpelan a todos los seres humanos de todos los tiempos, como individuos y como sociedades. Las respuestas nunca serán definitivas, sólo definitorias, porque modelan a cada una de las culturas, en sus diferentes épocas históricas y delimitan nuestra biografía personal.

Todo proyecto educativo está obligado a contemplarlas, tanto en el diseño curricular de cada programa, 
como en el contenido de los mismos, con el objeto de contribuir a que los estudiantes lleguen a desarrollarse en plenitud; por esto, la filosofía educativa del ITAM define desde el principio la noción en que se inspira:

Un concepto que entiende al ser humano como ser libre, como ser social comprometido en la elevación y el progreso humano y como ser llamado por vocación esencial a buscar la verdad y el bien. Toda educación, por tanto, debe tender a mejorar al ser humano mediante el enriquecimiento de sus mejores valores, la integración de su persona, la formación de su conciencia y el acrecentamiento de su capacidad de servicio. Asimismo, reconoce la obligación que adquiere respecto al desarrollo de la comunidad en que actúa, asumiendo su lealtad a México, a sus tradiciones y valores, entendidos como patrimonio colectivo. ${ }^{3}$

El maestro José Ramón Benito, director de la División Académica de Estudios Generales y Estudios Internacionales, explica que la genuina educación debe llevar al alumno a descubrir y reflexionar acerca de los valores desde los cuales vive un hombre:

La formación del hombre es la aspiración de todo humanismo y de toda cultura [...] la eficacia profesional

${ }^{3}$ http://www.itam.mx/es/acerca/mision/ mision.php y el desarrollo personal, lejos de ser cosas opuestas, resultan en realidad indisolubles. Y así, el desempeño de una actividad específica dentro de la sociedad no puede lograrse sino dentro de una auténtica formación humana [...] A partir de lo que un hombre piensa y de cómo ve el mundo, es que habrán de derivar sus acciones, sus afanes y sus actitudes. $\mathrm{Al}$ menos son sus convicciones más hondas las que lo animan y le dictan las normas o imperativos que dan sentido a su responsabilidad. Es en función de una cierta jerarquía de valores más o menos implícita, pero real, que un hombre vive. El concepto de hombre, de mundo y de vida que se tenga será lo que fundamenten un cierto modo de comportarse y de "justificar" los fines que se persigan y las técnicas profesionales que se utilicen. ${ }^{4}$

Aunque el ITAM plantea que la formación integral es quehacer de todas las entidades académicas, sin embargo, el Departamento de Estudios Generales tiene una responsabilidad sustancial, en tanto que busca la integración de la persona y su desarrollo social desde la perspectiva humanista fundamental. Por ello, se entiende a sí misma como la entidad académica más propiamente orientada a formar

${ }^{4}$ José Ramón Benito Alzaga, Estudios Generales en el ITAM, ponencia presentada en la Asamblea Nacional de ANFECA, julio de 1973, http://generales. itam.mx/docs/ESTUDIOS\%20GENERALES\%20 EN\%20ITAM.pdf. 
en la persona un tipo de cualidad que le permita su desarrollo completo y le capacite, para ubicarse significativa y responsablemente en el mundo a partir de una trayectoria histórica. ${ }^{5}$

Por otra parte, el Departamento es una entidad auténticamente interdisciplinaria, tanto por la variada formación de los profesores que lo constituyen, como por el contenido del área académica que, al centrarse en lo humano, comprende:

\section{1) Toda expresión del hombre cuya consideración intelectual no se limite exclusivamente al campo de los métodos y explicaciones de las ciencias particulares.}

2) A un conjunto de áreas específicas constituido por lo que podría seguirse llamando "humanidades": antropología, historia, literatura, arte, educación, religión y filosofía.

3) En lo académico, se busca abrir horizontes, ofrecer criterios para la integración y permitir la síntesis vital y personalísima de cada estudiante. ${ }^{6}$

Asimismo, debido a la dimensión histórica del hombre, tenemos que situarnos desde el presente para ver el pasado y proyectar el futuro. Las circunstancias presentes, es decir, fia.html.

${ }^{5}$ http://generales.itam.mx/contenido/filoso-

${ }^{6}$ Ibidem. nuestra época, se caracterizan por lo que algunos pensadores llaman el final de un tiempo. ${ }^{7}$ En el último siglo han ocurrido profundos cambios. La existencia humana se ha transformado de múltiples maneras, que incluyen las concepciones del mundo, del espacio, del tiempo, de la naturaleza $y$, por ende, las significaciones de la propia existencia. En este sentido, el papel que debe jugar la educación hoy es inédito, porque además de superar los errores del pasado, debe afrontar los retos del futuro.

\section{Introducción}

Los cursos de Problemas de la Civilización Contemporánea I y II pertenecen al conjunto de las siete materias obligatorias que imparte Estudios Generales en el ITAM. El Departamento define la temática general de estos cursos como: "Todas aquellas expresiones y manifestaciones del hombre o aspectos de la realidad actual que se prestan a la discusión".

${ }^{7}$ Cfr. José Ortega y Gasset, Pasado y porvenir para el hombre actual, Madrid, 1971, Revista de Occidente, t. IX, pp. 645-63; Eric Hobsbawm, Historia del siglo XX, 1998, Buenos Aires, Crítica-GrijalboMondadori, p. 13; Luis Villoro, Tres retos de la sociedad por venir. Justicia, democracia, pluralidad, 2009, México, Siglo XXI; Luis González, "El linaje de la cultura mexicana" en Vuelta, 1982, núm. 52, pp. 14-23, entre muchos otros.

${ }^{8}$ http://generales.itam.mx/contenido/filosofia.html 
En términos generales, se busca que el alumno analice los múltiples factores que han influido sobre la problemática contemporánea, para lo cual se recurre a las explicaciones de las diversas ciencias particulares; pero se busca presentarlos no sólo vinculados entre sí, sino interrelacionados con los aspectos humanos y con las serias implicaciones que pudieran tener en la dignidad del hombre. Los materiales que se analizan en ambos cursos son seleccionados por su actualidad, buscando siempre que los autores que los presentan sean de reconocida autoridad en su campo, independientemente de su postura ideológica. En casos de concepciones controvertidas, se presentan las más contrastantes a fin de que los alumnos tomen postura, dentro de un ambiente de pluralismo ideológico.

Con estas materias se intenta superar la fragmentación de saberes, la causalidad unilineal, la rigidez de ciertas lógicas y la reducción del conocimiento. Se procura construir una nueva imagen del mundo para comprender los problemas fundamentales, las cuestiones globales, situar los conocimientos parciales, relacionar los niveles locales, regionales, nacionales y mundiales. Además, se intenta desarrollar y enseñar métodos que permitan interpretar las relaciones mutuas y las influencias recíprocas entre las partes y el todo para entender la complejidad de la realidad, a fin de que los alumnos sean conscientes de los retos fundamentales e ineludibles frente a los cuales deben actuar con responsabilidad. Entre ellos, cabe mencionar la consecución de un desarrollo equitativo y sustentable que incorpore a los diversos grupos humanos y, al mismo tiempo, respete sus particularidades. En síntesis, se trata de formar a los alumnos para que puedan decidir con lucidez. ${ }^{9}$

\section{Objetivos}

\section{Docentes}

Problemas de la Civilización Contemporánea en realidad es una materia que se cursa en dos semestres y, como todas las materias de Estudios Generales, los objetivos tienen tres niveles: contenidos, destrezas mentales y responsabilidad social.

a) En el nivel de contenidos se busca dar una información que permita al estudiante la comprensión de ideas, problemas, hechos y sus relaciones, en la interrelación del pasado con el presente y hacia el futuro.

${ }^{9}$ ITAM, Taller de reflexión estratégica, División Académica de Estudios Generales y Estudios Internacionales, Jurica, febrero 2001, México, p. 5. 
b) En el nivel de destrezas mentales se busca dar al estudiante una formación que incremente su capacidad de razonamiento y expresión, su pensamiento crítico y su creatividad.

c) En el nivel de responsabilidad social se busca propiciar en los estudiantes la coherencia intelectualmente fundada entre el pensamiento y las actitudes, fomentando su capacidad de respuesta hacia el desarrollo de una sociedad más libre y más próspera y de un mayor progreso social". ${ }^{10}$

\section{Método de enseñanza}

Supone la participación de los estudiantes. Antes de cada lección el alumno debe leer y analizar los textos y materiales cuidadosamente seleccionados por los profesores del departamento. La clase se desarrolla conforme a los criterios del método mayeútico, semejante a la modalidad de seminario. El docente coordina la discusión grupal en la que los estudiantes reconstruyen el sentido del texto, clarifican su contenido y a medida que van expresándose ordenan, asimilan y paulatinamente descubren fia.html.

${ }^{10} \mathrm{http}: / /$ generales.itam.mx/contenido/filoso- su significado; así, pueden emitir juicios fundados y responsables sobre los tópicos estudiados.

\section{Diálogo}

Con el diálogo participativo los alumnos obtienen los siguientes resultados pedagógicos:

1. En el nivel de los conocimientos; gracias al diálogo el alumno afirma lo que comprendió de las fuentes originales, aclara los conceptos obscuros, asimila mejor las aportaciones de su profesor y de sus compañeros en un contexto vivo y concreto $\mathrm{y}$, así, se apropia de lo que aprende para iluminar su existencia personal y comunitaria.

2. En el ámbito de las destrezas, el diálogo ordenado y con rigor intelectual afirma las capacidades de análisis, síntesis, pensamiento crítico del alumno y le permite el desarrollo de capacidades mentales; favorece la madurez intelectual, lo hace capaz de pensar por sí mismo y sostener sus convicciones, que se ponen en duda para confirmarse o modificarse al analizar las discrepancias y argumentaciones, obligándolo a fundamentar sus propios juicios.

3. En términos de la responsabilidad social, el diálogo sitúa a los 
interlocutores, profesores y estudiantes, como personas comprometidas con sus juicios sobre el mundo, su sociedad y su historia.

Finalmente, los ensayos y exámenes buscan la integración de los aprendizajes adquiridos y habilidades desarrolladas.

\section{Estructura}

Los cursos de Problemas de la Civilización Contemporánea tienen una doble estructura, una temática y otra metodológica.

\section{Estructura metodológica}

Todos los temas tratados en los dos cursos, excepto la introducción llamada "Educación y Universidad" y la conclusión titulada "Educación y Desarrollo", cuentan con las siguientes partes: Introducción, Planteamientos, Áreas de Conflicto e Interpretaciones y Perspectivas.

De esta manera, cada tema está estructurado de acuerdo a los principios del método científico, con el objetivo de que los alumnos aprendan la manera de trabajar en la Universidad y en qué consiste el rigor académico; así, pueden elaborar sus juicios y respuestas de manera fundada y responsable.

\section{Introducción}

Contiene una serie de textos literarios, para que los estudiantes formulen preguntas acerca de los asuntos a tratar.

\section{Planteamientos generales}

Los textos que se leen están escritos por diversos autores, considerados expertos en esas materias, $\mathrm{y}$ en los que explican, definen conceptualmente y formulan teorías acerca de dichas cuestiones.

Áreas de conflicto

En esta sección se proponen algunos estudios de caso que presentan dichos problemas vivos en diversas realidades.

\section{Interpretaciones y perspectivas}

Donde los pensadores exponen, precisamente, diversas interpretaciones y perspectivas de lo que está sucediendo o podría pasar, así como algunas posibles soluciones.

\section{Estructura temática}

En este apartado se analiza las preguntas fundamentales que sirven de hilo conductor para unir las diversas temáticas y su tratamiento, con el auxilio y la integración de diversas ciencias, a fin de permitir que cada 
estudiante llegue a entenderse a sí mismo, comprenda su mundo y sea capaz de asumir libre y racionalmente su compromiso en la construcción del porvenir.

Los temas tratados en el primer semestre son: 1) Educación y Universidad; 2) Civilización y cultura; y, 3) Individuo y sociedad. En el segundo semestre se tratan: 4) Población y hábitat; y, 5) El hombre como creador y portador de cultura. Además, hay una "Introducción general" a los dos cursos, que se estudia en Problemas I $\mathrm{y}$, una conclusión general situada al final de Problemas II, llamada "Educación y Desarrollo".

La estructura sigue un orden pedagógico, tanto por el nivel de dificultad como por la gradualidad y los requerimientos de los contenidos temáticos. Por ejemplo, no se pueden entender los problemas de Población y hábitat sin haber estudiado las diversas visiones culturales y sistemas sociales, económicos y políticos implicados.

Todos ellos, además de la importancia propia de cada contenido, cumplen con el primer objetivo general arriba mencionado, proporcionar al estudiante una información que le permita comprender el mundo en que vive y comprenderse en él.

\section{Selección de textos y materiales de estudio}

La estructura de los cursos y la selección de los materiales es una tarea de reflexión y búsqueda siempre inacabada que involucra de manera distinta a los profesores del Departamento. Para cada grupo de materias se cuenta con un comité que se reúne semanalmente para analizar y discutir sobre los acontecimientos mundiales, los nuevos materiales encontrados y para evaluar el funcionamiento de los materiales, así como los comentarios de los alumnos y profesores que imparten la materia. Diversos materiales se han incorporado por sugerencia de alumnos y profesores. Los criterios para seleccionar los materiales de los cursos son:

1. Actualidad, entendida como lo que es actuante, es decir, lo que afecta seriamente al desarrollo integral del hombre; no se trata sólo de buscar lo nuevo por ser novedoso o por estar a la moda si sólo es un tema coyuntural.

2. Textos y materiales apropiados para ser "recortados", sin perder las ideas principales. Se busca que tanto por la longitud como por el nivel de dificultad, la exigencia de que los alumnos lean el texto completo sea realmente posible de cumplir. 
3. Autores reconocidos en su campo de estudio y que muestren una perspectiva de la complejidad del problema, con un lenguaje accesible para los universitarios, no sólo para los especialistas.

4. Pluralidad de posturas ideológicas. Se busca exponer posiciones contrastantes, para que los alumnos puedan formar su criterio y elegir racional y responsablemente.

5. Análisis de en qué parte de la estructura del curso se debe colocar el material y en qué orden. Se trata de respetar la estructura, mientras se vea que funciona para explicar al hombre y al mundo y plantear futuros posibles.

La preparación de los cursos y materiales es una labor lenta y compleja, que funciona gracias a la participación de muchas personas interesadas en construir una nueva imagen del cosmos y comprender los problemas fundamentales del hombre en su tarea de construir mundos y construirse a sí mismo.

\section{Problemas de La CIVILIZACIÓN CONTEMPORÁNEA I}

\section{- Educación y universidad}

Tiene como objetivos específicos introducir a los alumnos recién llegados a la universidad, a la filosofía, la misión y los principios del ITAM, al método del diálogo crítico y participativo de todas las materias del Estudios Generales, así como a los problemas de la civilización contemporánea. Todo esto, para que el estudiante vea que desde la universidad se piensan los problemas y se aportan soluciones. Creemos que es la misión de la universidad, tal y como la define Carlos de la Isla: "la universidad es la conciencia crítica de la sociedad":

Conciencia crítica de la sociedad significa que la misión de la Universidad es pensar (la investigación no es otra cosa que pensar al mundo y a nosotros en el mundo), enseñar a pensar (y ésta es la esencia de la educación universitaria), transmitir $\mathrm{y}$ acrecentar el pensamiento (en esto consiste la extensión de la cultura). La Universidad como conciencia crítica de la sociedad debe conocer la realidad social en su totalidad, esta es la materia de su pensamiento, pero esta acción reflexiva sobre la sociedad no termina en el pensamiento, ha de juzgarla y con actitud crítica, denunciar, anunciar, inventar. ${ }^{11}$

\section{- Civilización y cultura}

Las inquietudes que trata este apartado se resumen en las siguientes cuestiones: ¿Las diferencias entre los hombres son esenciales o accidenta-

${ }^{11}$ Carlos De la Isla, La Universidad: conciencia crítica, México, ITAM, 1991, en http://generales.itam.mx/docs/UNIVERSIDADCONCIENCIA\%20CRITICA.pdf 
les? ¿Qué tenemos en común a pesar de nuestras diferencias? ¿Se justifican actitudes como el etnocentrismo, racismo, segregación o dominio cultural? ¿Cómo vemos a los otros? ¿Cómo los otros nos ven a nosotros? ¿Cómo y de qué maneras nos influimos mutuamente? ¿Qué implican planteamientos tales como homogeneidad y pluralidad? ¿Es posible la tolerancia? ¿En qué aspectos? Y, en última instancia, ¿podremos vivir juntos? Es decir, se trata de reflexionar sobre nuestro destino común y nuestra responsabilidad en la construcción de la humanidad.

\section{- Individuo y sociedad}

Los temas que se tratan en este apartado interpelan a los estudiantes en los siguientes aspectos: ¿Cuáles son las relaciones entre el individuo y la sociedad? ¿La sociedad es una suma de individuos o es una comunidad? ¿Si el hombre es producto de la sociedad, cómo puedo llegar a ser yo mismo? ¿La sociedad me determina o me condiciona? ¿Existe una armonía o una tensión entre los distintos ámbitos de la sociedad, tales como la economía, la política, la familia? ¿Por qué estoy obligado a decidir? ¿Existen sistemas políticos, económicos o sociales perfectos? ¿Tengo responsabilidades sociales? ¿Qué es el cambio social? ¿Cuál es el papel del indivi- duo al respecto? Se trata de analizar críticamente los defectos de todos los sistemas políticos y sociales en relación con sus implicaciones en el desarrollo humano de todos los hombres y de todo el hombre.

\section{Problemas de la Civilización CONTEMPORÁNEA II}

\section{- Población y Hábitat}

Tal parece que la cultura de los próximos años será ecológica, si queremos sobrevivir; sin embargo, las posturas existentes son muy diversas y contradictorias por lo que se requiere de una reflexión crítica y seria de todas sus implicaciones poblacionales, sociales, económicas, políticas, éticas, humanas. Por ello, en este apartado se plantean interrogantes como las que aquí se enumeran: ¿Qué es la naturaleza? ¿Qué relaciones existen entre el hombre y la naturaleza? ¿Cuáles son las razones para conservar a la naturaleza, con el hombre o contra el hombre? ¿Qué relaciones existen entre población y recursos? ¿Cuánto es suficiente? ¿Qué significa la sustentabilidad? ¿Todas las formas de vida, producción, distribución y consumo son sustentables? ¿Qué es el calentamiento global? ¿Qué es la vida humana y cuál es su valor? 
- El hombre como creador y portador de cultura

En este apartado se busca que el estudiante se descubra a sí mismo, en su integralidad, que vea las dimensiones de lo humano que se manifiestan en la cultura; que analice los ámbitos de su hacer y obrar, a fin de que asuma con lucidez su propia vida y las circunstancias y retos que le toca vivir. Las preguntas que lo llevan a este análisis pueden sintetizarse: ¿Qué es la persona? ¿Qué significa la acción libre? ¿Por qué el hombre debe construirse a sí mismo? ¿Qué es lo específicamente humano? ¿Por qué el hombre necesita contemplar y reflexionar? ¿Qué importancia tienen las consideraciones éticas, estéticas, técnicas y científicas? ¿Por qué aprender a ponerme en el lugar de otras personas? ¿Qué permite imaginar y crear? En resumen, se trata de asumir el significado y sentido de la propia existencia, de capacitar al estudiante para que pueda desarrollar de manera coherente y no exclusivamente racional su síntesis vital.

\section{- Educación y desarrollo}

A manera de conclusión de los dos cursos se llega a la cuestión de la responsabilidad social que tienen los universitarios, no sólo en su ejercicio profesional, sino también como un compromiso para el logro del desarrollo integral que implican todos los ámbitos de la persona y las sociedades, tal y como los cursos intentan mostrar. Se trata de reflexionar sobre el bien común y la justicia. Pero, a diferencia de las discusiones sobre este tema en los países desarrollados de Occidente, como bien dice Villoro, en países como México, en lugar de partir del consenso para fundar la justicia, tenemos que "partir de su ausencia... de la percepción de la injusticia real para proyectar lo que podría remediarla". ${ }^{12}$ Se trata de mostrar que a pesar de todo, el futuro no está determinado, sino que es tarea en cuya construcción estamos obligados a participar.

\section{Reflexiones finales a manera de conclusión}

Los cursos de Problemas de la Civilización Contemporánea del ITAM contribuyen a la formación integral de los alumnos mediante contenidos interdisciplinarios que integran las ciencias particulares en una perspectiva humanista.

Así, todos los temas tratados se unen en la preocupación por el hombre, en este conocimiento siempre antiguo y siempre nuevo del ser humano, de su integralidad, de sus obras,

${ }^{12}$ Luis Villoro, Tres retos de la sociedad por venir. Justicia, democracia, pluralidad, 2009, México, Siglo XXI, p. 12-3. 
de sus motivaciones; de las formas, características y configuración de sus relaciones; sus coordenadas, su finitud, su trascendencia, sus valores; sus posibilidades de acción para transformar la naturaleza; el medio que lo rodea, tanto físico como sociocultural; los riesgos que ocasiona con las modificaciones de su ambiente ecológico y social; más aún, su capacidad de transformarse a sí mismo, cambiando el curso de la historia y de los condicionantes de su conducta y acción, enjuiciando así la validez del "progreso".

El método docente que se aplica en estos cursos subraya la importancia de la reflexión crítica, ordenada y fundamentada de cada estudiante. Se entiende que la formación integral es principalmente una educación para la libertad y su correlato en la responsabilidad personal y social.

Este es el motivo por el cual los cursos no dan respuestas, sólo plantean preguntas y señalan caminos posibles, aunque no todos ellos deseables, que interpelan al alumno y lo urgen a tomar postura y comprometerse. Porque la auténtica libertad, como dice José Ramón Benito:

Sólo puede darse a partir de una coherencia entre el pensamiento y la vida, las convicciones y las actitudes, coherencia que sólo puede ser intelectualmente fundada por una profunda revisión que permita tomar una sólida posición frente al sistema de ideas que se sustenta y que haga patente su reclamo para la vida. ${ }^{13}$

La síntesis final y vital es obra del propio estudiante. En última instancia, el uso que los estudiantes puedan hacer de estos conocimientos y destrezas depende de la responsabilidad que cada uno quiera asumir.
${ }^{13}$ José Ramón Benito Alzaga, Notas sobre el sentido social de la formación humanista en el ITAM, México, ITAM, http://generales.itam.mx/ docs/FORMACION\%20HUMANISTA.pdf 